\title{
Polarization and Wide Incident Angle Insensitive Metal Free Superwideband Absorber For Terahertz and Infrared Spectrum
}

SARTHAK SINGHAL ( $\nabla$ sarthak.ece@mnit.ac.in )

Malaviya National Institute of Technology Jaipur https://orcid.org/0000-0002-1160-6150

\section{Research Article}

Keywords: Absorption bandwidth, infrared frequency spectrum, metal free absorber, superwideband absorber, terahertz applications, wide incident angle insensitive

Posted Date: March 22nd, 2022

DOI: https://doi.org/10.21203/rs.3.rs-1366608/v2

License: @ (i) This work is licensed under a Creative Commons Attribution 4.0 International License. Read Full License 


\section{Abstract}

A circular slot loaded octagonal metal free graphite based absorber for superwideband applications at terahertz and infrared spectrum is proposed. The absorber comprises a circular slot loaded octagonal graphite slab at the top layer, silicon dioxide as substrate and square graphite slab as the bottom layer. This absorber is operating over a frequency spectrum of $6 \mathrm{THz}$ to more than $80 \mathrm{THz}$ (ratio bandwidth of >12:1 and fractional bandwidth of $>172.093 \%$ ) for absorption more than $90 \%$ with unity peak absorption. The absorber dimensions are $5 \mu \mathrm{m} \times 5 \mu \mathrm{m} \times 7.04 \mu \mathrm{m}$. The thickness of the substrate is only $\sim \lambda \mathrm{o} / 26$ and the periodicity of absorber is $\sim \lambda \mathrm{o} / 10$ at $6 \mathrm{THz}$. The performance of the absorber is found to be insensitive to changes in polarization angle $(\Phi)$. Furthermore, the performance of the absorber is observed to be unaffected by incident angle $(\theta)$ variations from $0^{\circ}$ to $60^{\circ}$. The metal-free geometry along with insensitiveness to $\Phi$ and $0^{\circ} \leq \theta \leq 60^{\circ}$ makes the proposed absorber suitable for compact terahertz/infrared micro/nanoscale systems.

\section{Introduction}

Terahertz ( $\mathrm{THz}$ ) spectrum has received much interest from academia and industry in the last two decades for its vast applications in communication, imaging, medical, radar, sensing, spectroscopy, and other fields. The field of absorbers has been determined to be the most practical use of the $\mathrm{THz}$ spectrum. Researchers have recently reported several single and multilayered geometry-based terahertz absorbers having narrowband, multi-band, wideband, and frequencydependent reconfigurable performance [1-34]. Different techniques leading to the excitation of multiple resonances are used to design multi-band absorbers. The merging of these multiple resonances leads to wideband performance. Multilayered or multiple stacked systems provide wide bandwidth; however, they are challenging to implement. The easy to implement single layer structures provide limited operating frequency range. Therefore, a major challenge is to design a broadband absorber having single layer of substrate without any stacking of metallic/graphene layers.

Another challenge faced during absorber designing is the limited performance at higher frequencies due to the metals' limited electrical properties and high-temperature sensitivity, which is mainly responsible for incident power absorption in metal/dielectric multi-layered absorbers $[27,28]$. Few researchers have reported absorbers based on vanadium oxide, a temperature-sensitive \& phase change material [21, 29]. They provide desired wideband performance even at high temperatures, but these high temperatures affect the functioning of other nano-scale components. All the abovediscussed broadband absorbers have complex fabrication techniques, thick geometries, and temperature-dependent performance. Thus, there is a need to design broadband absorbers whose fabrication is easy, the substrate thickness is negligible, and the performance is temperature independent. According to the available literature, metal-free absorbers like graphite/dielectric-based absorbers and semiconductor grating-based absorbers have provided temperatureindependent performance [26, 30,31]. The major reason for the usage of graphite in flexible electronics components like THz antennas and high-frequency absorbers is its better temperature stability [21]. These broadband metal-free absorbers can cover only a small portion of the terahertz spectrum. The future requirements require the coverage of both terahertz and infrared spectrum.

This paper presents the design and analysis of a metal-free graphite/dielectric-based super wideband absorber. The combination of circular slot-loaded octagonal graphite slab, silicon dioxide dielectric material, and square graphite slab resulted in an operating frequency range from $6 \mathrm{THz}$ to more than $80 \mathrm{THz}$ with the absorption of more than $90 \%$ for the normal incidence of electromagnetic waves. In two frequency bands of 7.85-13.05 THz and 22.47 THz to more than 80 $\mathrm{THz}$, the absorption is found to be more than $95 \%$. In the frequency spectrum of $25.35-28.71 \mathrm{THz}, 44.68-74.65 \mathrm{THz}$, and $79.52 \mathrm{THz}$ to more than $80 \mathrm{THz}$, the absorption is more than $99 \%$. The proposed absorber's overall volume is significantly lesser than several dielectric, metamaterial, and grating-based structures. The advantageous features of planar metal-free geometry with miniaturized dimensions, low volume, insensitivity to polarization angle, and wide 
incident angle range make this absorber compatible with the future nanoscale super wideband terahertz and infrared systems.

\section{li. Absorber Design}

The proposed antenna geometry, shown in Fig. 1, is a three layered structure. In the proposed absorber, a $1.54 \mu \mathrm{m}$ thick silicon dioxide substrate is sandwiched between two graphite slabs. The top graphite slab is octagonal in shape. It is loaded with a central circular slot. During the simulation of the proposed absorber structure by using finite integration technique (FIT)-based CST Microwave Studio (CST MWS), periodic boundaries are fixed along x-and y-axis. Along the zaxis i.e. transmission direction, open boundary conditions are fixed. This absorber is designed in three stages i.e. initially only graphite ground is analyzed. In second stage, the combination of graphite ground and substrate is analyzed. In the last stage, the circular slot loaded octagonal graphite, substrate material and graphite ground plane are analyzed together.

\section{lii. Results And Discussion}

The magnitudes of reflectance $\left(S_{11}\right)$ and transmittance $\left(S_{21}\right)$ for all absorber designing stages are shown in Fig. 2 . It is observed that for each stage the magnitude of transmittance is almost zero i.e. $\left|S_{21}\right| \cong 0$. The reflectance for first stage is decreasing linearly from 1 to 0.7 in the frequency range of 1 to $80 \mathrm{THz}$ without any resonance. For second stage, two resonance at the frequencies of 22.79 and $69.85 \mathrm{THz}$ with $\left|\mathrm{S}_{11}\right|$ of 0.55 and 0.23 are observed. In the last stage, the reflectance is observed to be sharply decreasing from 1 to 0.1 with three resonances. For the frequency range of 6.33 $\mathrm{THz}$ to more than $80 \mathrm{THz}$, the reflectance magnitude is less than 0.3. By using these values of reflectance and transmittance in the absorption equation, $A=1-\left|S_{11}\right|^{2}-\left|S_{21}\right|^{2}$ the values of absorption are calculated for each designing stage. Since $\left|S_{21}\right|=0$, therefore the above equation is reduced to $A=1-\left|S_{11}\right|^{2}$. The absorption values for each designing stage are shown in Fig. 2 (c). For first stage, a maximum absorption of $50 \%$ is achieved. In second stage, two peaks having absorption of $70 \%$ and $95 \%$ are observed at resonance frequencies. In the last stage, absorption of more than $90 \%$ is achieved in the frequency spectrum of $6 \mathrm{THz}$ to more than $80 \mathrm{THz}$. Figure 3 illustrates a good agreement between the absorption in both modes i.e. transverse electric (TE) and magnetic (TM) for the proposed absorber.

To analyze the polarization sensitiveness of this absorber, the polarization angle $(\Phi)$ is varied from $0^{\circ}$ to $90^{\circ}$. Due to the geometrical symmetry, this absorber is insensitive to the variations in polarization angle as shown in Fig. 4.

Table 1 illustrates that the proposed absorber has widest bandwidth ( $6 \mathrm{THz}$ to more than $80 \mathrm{THz}$ ) along with minimum volume of $176 \mu \mathrm{m}^{3}$ among the compared structures. The proposed absorber is covering terahertz frequency spectrum from 6-10 THz and infrared region from $10 \mathrm{THz}$ to more than $80 \mathrm{THz}$ whereas the operating band of other absorbers is covering only a narrow portion of the terahertz spectrum. In addition to this, this absorber is polarization angle and wide incident angle insensitive. 
Table 1

Comparison of proposed absorber with other absorber structures

\begin{tabular}{|c|c|c|c|c|c|c|c|c|}
\hline \multirow[t]{2}{*}{ Reference } & \multirow[t]{2}{*}{ Geometry } & \multirow[t]{2}{*}{ Material } & \multirow[t]{2}{*}{$\begin{array}{l}\text { Unit cell } \\
\text { dimensions } \\
(\mu \mathrm{m} \times \mu \mathrm{m} \times \\
\mu \mathrm{m})\end{array}$} & \multirow[t]{2}{*}{$\begin{array}{l}\text { Absorption } \\
\text { operating } \\
\text { range in } \\
\text { THz / } \\
\text { (Absorption } \\
\text { criteria in } \\
\% \text { ) }\end{array}$} & \multirow[t]{2}{*}{$\%$ BW } & \multirow[t]{2}{*}{$\begin{array}{l}\text { Polarization } \\
\text { insensitivity }\end{array}$} & \multicolumn{2}{|c|}{$\begin{array}{l}\text { Incident } \\
\text { Angle } \\
\left(\theta^{\circ}\right) \\
\text { (for A> } \\
90 \%)\end{array}$} \\
\hline & & & & & & & TE & TM \\
\hline [6] & $\begin{array}{l}\text { Multiple } \\
\text { square } \\
\text { patches } \\
\text { resonators }\end{array}$ & $\begin{array}{l}\text { Copper and } \\
\text { SiO2 }\end{array}$ & $20 \times 20 \times 0.7$ & $\begin{array}{l}6.23-7.08 \\
\mathrm{THz} /(80) \\
6.24-7.04 \\
\mathrm{THz} /(90)\end{array}$ & $\begin{array}{l}12.772 \\
12.048\end{array}$ & yes & - & - \\
\hline [7] & $\begin{array}{l}\text { Multiple } \\
\text { stacked bars } \\
\text { resonators }\end{array}$ & $\begin{array}{l}\text { Polyimide and } \\
\text { Gold bars }\end{array}$ & Not available & $\begin{array}{l}0.8-1.368 \\
\mathrm{THz} /(80) \\
0.8 \text { to } 1.28 \\
\mathrm{THz} /(90)\end{array}$ & $\begin{array}{l}52.399 \\
46.154\end{array}$ & no & 40 & 40 \\
\hline [8] & $\begin{array}{l}\text { Hybrid } \\
\text { Structure }\end{array}$ & $\begin{array}{l}\text { Polyimide and } \\
\text { Gold }\end{array}$ & $376 \times 376$ & $\begin{array}{l}0.13 \\
\mathrm{THz}(80) \\
0.22-0.33 \\
\mathrm{THz} /(90)\end{array}$ & $\begin{array}{l}0 \\
40\end{array}$ & Yes & 45 & 45 \\
\hline [9] & $\begin{array}{l}\text { Sectional } \\
\text { asymmetric } \\
\text { structure }\end{array}$ & Au and Si3N4 & $19.4 \times 19.4$ & $\begin{array}{l}4.6-5.1 \\
\mathrm{THz} /(80)\end{array}$ & 10.309 & No & 30 & 30 \\
\hline [10] & $\begin{array}{l}\text { Fractal } \\
\text { cross } \\
\text { resonators }\end{array}$ & $\begin{array}{l}\text { Polyimide and } \\
\text { Gold }\end{array}$ & $40 \times 40 \times 11.24$ & $\begin{array}{l}3.01-4.84 \\
\mathrm{THz} /(80)\end{array}$ & 46.624 & - & 45 & 45 \\
\hline [11] & $\begin{array}{l}\text { Stacked } \\
\text { cross } \\
\text { resonators }\end{array}$ & $\begin{array}{l}\text { Parylene, Gold, } \\
\text { Aluminium } \\
\text { and Silicon }\end{array}$ & $80 \times 80 \times 12$ & $\begin{array}{l}7.1-8 \mathrm{THz} / \\
(80)\end{array}$ & 11.921 & - & - & - \\
\hline [12] & $\begin{array}{l}\text { Nested } \\
\text { circular } \\
\text { rings }\end{array}$ & $\begin{array}{l}\text { Copper and } \\
\text { polyimide }\end{array}$ & $30 \times 30 \times 10.2$ & $\begin{array}{l}1.6-2.6 \\
\mathrm{THz} /(80) \\
0.896 \mathrm{THz} \\
/(90)\end{array}$ & $\begin{array}{l}47.619 \\
0\end{array}$ & Yes & 60 & 60 \\
\hline [13] & $\begin{array}{l}\text { Truncated } \\
\text { pyramid } \\
\text { structure }\end{array}$ & $\begin{array}{l}\text { SU8 polymer } \\
\text { and gold }\end{array}$ & $95 \times 95 \times 21$ & $\begin{array}{l}0.75-1.5 \\
\mathrm{THz} /(80)\end{array}$ & 66.667 & Yes & 30 & 70 \\
\hline [14] & $\begin{array}{l}\text { Grating } \\
\text { structure }\end{array}$ & Doped Silicon & $100 \times 100 \times 500$ & $\begin{array}{l}0.58-2.58 \\
\mathrm{THz} /(90)\end{array}$ & 126.582 & Yes & 45 & 55 \\
\hline [15] & $\begin{array}{l}\text { Multiple I- } \\
\text { shaped } \\
\text { strips }\end{array}$ & $\begin{array}{l}\text { Polyimide and } \\
\text { gold }\end{array}$ & $78 \times 78 \times 8.5$ & $\begin{array}{l}0.87-0.97 \\
\mathrm{THz} /(80)\end{array}$ & 10.87 & Yes & 60 & 60 \\
\hline [16] & $\begin{array}{l}\text { Pythagorean } \\
\text { tree fractal } \\
\text { structure }\end{array}$ & $\begin{array}{l}\text { Polyimide and } \\
\text { gold }\end{array}$ & $69 \times 69 \times 22.2$ & $\begin{array}{l}7.5-10 \\
\mathrm{THz} /(80)\end{array}$ & 28.571 & No & 0 & 0 \\
\hline [17] & $\begin{array}{l}\text { Multiple } \\
\text { diamond } \\
\text { array }\end{array}$ & $\begin{array}{l}\text { Dielectric and } \\
\text { diamond }\end{array}$ & $170 \times 170 \times 310$ & $\begin{array}{l}1.3 \mathrm{THz} / \\
(90)\end{array}$ & 95.65 & No & 45 & 40 \\
\hline
\end{tabular}




\begin{tabular}{|c|c|c|c|c|c|c|c|c|}
\hline \multirow[t]{2}{*}{ Reference } & \multirow[t]{2}{*}{ Geometry } & \multirow[t]{2}{*}{ Material } & \multirow[t]{2}{*}{$\begin{array}{l}\begin{array}{l}\text { Unit cell } \\
\text { dimensions }\end{array} \\
(\mu \mathrm{m} \times \mu \mathrm{m} \times \\
\mu \mathrm{m})\end{array}$} & \multirow[t]{2}{*}{$\begin{array}{l}\text { Absorption } \\
\text { operating } \\
\text { range in } \\
\text { THz / } \\
\text { (Absorption } \\
\text { criteria in } \\
\% \text { ) }\end{array}$} & \multirow[t]{2}{*}{$\%$ BW } & \multirow[t]{2}{*}{$\begin{array}{l}\text { Polarization } \\
\text { insensitivity }\end{array}$} & \multicolumn{2}{|c|}{$\begin{array}{l}\text { Incident } \\
\text { Angle } \\
\left(\theta^{\circ}\right) \\
(\text { for } A> \\
90 \%)\end{array}$} \\
\hline & & & & & & & TE & TM \\
\hline \multirow[t]{2}{*}{ [18] } & \multirow[t]{2}{*}{$\begin{array}{l}\text { Slot loaded } \\
\text { rectangular } \\
\text { structure }\end{array}$} & \multirow[t]{2}{*}{$\begin{array}{l}\text { Metal and } \\
\text { Dielectric }\end{array}$} & \multirow[t]{2}{*}{$88 \times 88 \times 14$} & $\begin{array}{l}1.3-2.7 \\
\mathrm{THz} /(90)\end{array}$ & \multirow[t]{2}{*}{70} & \multirow[t]{2}{*}{ No } & \multirow[t]{2}{*}{-} & \multirow[t]{2}{*}{-} \\
\hline & & & & $\begin{array}{l}1.24-2.86 \\
\mathrm{THz} /(50)\end{array}$ & & & & \\
\hline [19] & $\begin{array}{l}\text { three-layer } \\
\text { structure, } \\
\text { comprising } \\
\text { square-, } \\
\text { cross-, and } \\
\text { circular- } \\
\text { shaped }\end{array}$ & $\begin{array}{l}\text { Metal, } \\
\text { dielectric and } \\
\text { grapheme }\end{array}$ & $58 \times 58 \times 50$ & $\begin{array}{l}0.55-3.12 \\
\mathrm{THz} /(90)\end{array}$ & 140 & Yes & 15 & 45 \\
\hline [20] & $\begin{array}{l}\text { square ring } \\
\text { and multiple } \\
\text { T-shaped } \\
\text { resonators }\end{array}$ & $\begin{array}{l}\text { Metal and } \\
\text { dielectric }\end{array}$ & $120 \times 120 \times 22$ & $\begin{array}{l}4.904- \\
6.632 \mathrm{THz} \\
/(90)\end{array}$ & 29.95 & Yes & 0 & 0 \\
\hline [21] & $\begin{array}{l}\text { L shaped } \\
\text { resonators }\end{array}$ & $\begin{array}{l}\text { VO2 and } \\
\text { dielectric }\end{array}$ & $50 \times 50 \times 26$ & $\begin{array}{l}1.2-3.2 \\
\mathrm{THz} /(90)\end{array}$ & 90.90 & No & 50 & 50 \\
\hline [22] & $\begin{array}{l}\text { Cross } \\
\text { shaped } \\
\text { resonators }\end{array}$ & $\begin{array}{l}\text { Metal and } \\
\text { dielectric }\end{array}$ & $225 \times 225 \times 325$ & $\begin{array}{l}0.65-2.45 \\
\mathrm{THz} /(90)\end{array}$ & 116.12 & Yes & 40 & 40 \\
\hline [23] & $\begin{array}{l}\text { Rectangular } \\
\text { ring loops }\end{array}$ & $\begin{array}{l}\text { Metal,dielectric } \\
\text { and nitride }\end{array}$ & $70 \times 70 \times 24$ & $\begin{array}{l}1.17-2.99 \\
\mathrm{THz} /(90)\end{array}$ & 87.5 & Yes & 30 & 30 \\
\hline [24] & $\begin{array}{l}\text { wheel hub- } \\
\text { like }\end{array}$ & Metal & $200 \times 200 \times 90$ & $\begin{array}{l}\text { 1.6-5 THz / } \\
(90)\end{array}$ & 103 & Yes & 45 & 70 \\
\hline [25] & $\begin{array}{l}\text { Multiple } \\
\text { circular } \\
\text { resonators }\end{array}$ & $\begin{array}{l}\text { Metal, } \\
\text { dielectric and } \\
\text { grapheme }\end{array}$ & $80 \times 24 \times 28$ & $\begin{array}{l}1.6-3.2 \\
\mathrm{THz} /(90)\end{array}$ & 63.91 & No & 50 & - \\
\hline [26] & Annular ring & $\begin{array}{l}\text { Dielectric and } \\
\text { graphite }\end{array}$ & $85 \times 85 \times 50.2$ & $\begin{array}{l}0.65-3.03 \\
\mathrm{THz} /(90)\end{array}$ & 129.34 & Yes & 50 & 50 \\
\hline $\begin{array}{l}\text { Proposed } \\
\text { Absorber }\end{array}$ & $\begin{array}{l}\text { Octagonal } \\
\text { Annular } \\
\text { Ring }\end{array}$ & $\begin{array}{l}\text { Dielectric and } \\
\text { graphite }\end{array}$ & $5 \times 5 \times 7.04$ & $\begin{array}{l}6 \mathrm{THz} \text { to } \\
\text { more than } \\
80 \mathrm{THz} \\
\text { (90)/ } 4.8 \\
\text { THz to } \\
\text { more than } \\
80 \mathrm{THz} \\
\text { (80) }\end{array}$ & $\overrightarrow{172.093}$ & Yes & 60 & 60 \\
\hline
\end{tabular}

\section{Iv. Conclusion}

A superwideband absorber based on dielectric and graphite material for operation at terahertz and infrared frequency spectrum is investigated. It has an operating bandwidth of more than $74 \mathrm{THz}$ starting from $6 \mathrm{THz}$ for absorption more than $90 \%$. Due to the presence of only graphite as conducting material, this absorber will be insensitive to the 
temperature variations. Since the absorber geometry is symmetrical, it has insensitiveness to polarization angles. It is insensitive to a wide range of incident angle variations upto $60^{\circ}$.

\section{Declarations}

* Ethics approval: Not Applicable

* Consent to participate: Not applicable

* Consent for publication: Not applicable

* Availability of data and materials: Data sharing not applicable to this article as no datasets were generated or analysed during the current study.

* Competing interests: The author has no relevant financial or non-financial interests to disclose.

* Funding: The authors declare that no funds, grants, or other support were received during the preparation of this manuscript.

* Authors' contributions: The sole author Sarthak Singhal has contributed to the study conception, design, material preparation, data collection, analysis and writing of the manuscript.

* Acknowledgements: Not applicable

* Authors' information (optional).

Please include the sub-sections below of Compliance with Ethical Standards section.

* Disclosure of potential conflicts of interest: The author has no relevant financial or non-financial interests to disclose.

* Research involving Human Participants and/or Animals: Not applicable

* Informed consent: Only single author is there

\section{References}

1. A. Mohanty, O. Acharya, B. Appasani, and S. Mohapatra, "A multi-band terahertz metamaterial absorber based on a $\Pi$ and U-shaped structure," Photonics and Nanostructures - Fundamentals and Applications, vol. 32, pp. 74-80, 2018.

2. R. M. H. Bilal, M. A. Baqir, P. K. Choudhury, M. Karaaslan, M. M. Ali, O. AltIntas, A. A. Rahim, E. Unal, and C. Sabah, "Wideband Microwave Absorber Comprising Metallic Split-Ring Resonators Surrounded With E-Shaped Fractal Metamaterial," IEEE Access, vol. 9, pp. 5670-5677, 2021.

3. M. R. Soheilifar and R. A. Sadeghzadeh, Design, fabrication and characterization of stacked layers planar broadband metamaterial absorber at microwave frequency," AEU-Int. J. Electron. Commun., vol. 69, no. 1, pp. 126_132, Jan. 2015.;

4. D.-E.Wen, X. Huang, L. Guo, H. Yang, S. Han, and J. Zhang, Quadrupleband polarization-insensitive wide-angle metamaterial absorber based on multi-layer structure," Optik, vol. 126, nos. 9_10, pp. 1018_1020,May 2015.

5. Zhao $\mathrm{J}$ and Cheng $\mathrm{Y}$ (2016) A high-efficiency and broadband reflective $90^{\circ}$ linear polarization rotator based on anisotropic metamaterial. Applied Physics B: Photophysics and Laser Chemistry 255, 1-7.

6. Y. Cheng, Y. Nie, and R. Gong, "A polarization-insensitive and omnidirectional broadband terahertz metamaterial absorber based on coplanar multi-squares films," Opt. Laser Technol.48, 415-421 (2013). 
7. S. Liu, H. Chen, and T. J. Cui, “A broadband terahertz absorber using multi-layer stacked bars,” Appl. Phys. Lett.106(15), 151601 (2015).

8. Y. L. U. Uying, J. L. I. Ining, S. H. Z. Hang, and J. I. S. Un, “Polarization-insensitive broadband terahertz metamaterial absorber based on hybrid structures," Appl. Opt.57(21), 6269-6275 (2018).

9. C. Gong, M. Zhan, J. Yang, Z. Wang, H. Liu, Y. Zhao, and W. Liu, "Broadband terahertz metamaterial absorber based on sectional asymmetric structures," Sci. Rep.6(1), 32466-32473 (2016).

10. M. Kenney, J. Grant, Y. D. Shah, I. Escorcia-Carranza, M. Humphreys, and D. R. S. Cumming, “Octave-Spanning Broadband Absorption of Terahertz Light Using Metasurface Fractal-Cross Absorbers," ACS Photonics4(10), 26042612 (2017).

11. D. Jia, J. Xu, and X. Yu, "Ultra-broadband terahertz absorption using bi-metasurfaces based multiplexed resonances," Opt. Express26(20), 26227-26234 (2018).

12. W. Pan, X. Yu, J. Zhang, and W. Zeng, "A Novel Design of Broadband Terahertz Metamaterial Absorber Based on Nested Circle Rings," IEEE Photonics Technol. Lett.28(21), 2335-2338 (2016).

13. J. Zhu, Z. Ma, W. Sun, F. Ding, Q. He, L. Zhou, and Y. Ma, "Ultra-broadband terahertz metamaterial absorbear," Appl. Phys. Lett.105(2), 021102 (2014).

14. Y. Peng, X. Zang, Y. Zhu, C. Shi, L. Chen, B. Cai, and S. Zhuang, "Ultra-broadband terahertz perfect absorber by exciting multi-order diffractions in a double-layered grating structure," Opt. Express23(3), 2032 (2015).

15. L. Huang, D. R. Chowdhury, S. Ramani, M. T. Reiten, S. Luo, A. J. Taylor, and H. Chen, "a broad and flat high absorption band," Opt. Lett. 37(2), 154-156 (2012).

16. R. M. H. Bilal, M. A. Naveed, M. A. Baqir, M. M. Ali, and A. A. Rahim, “Design of a wideband terahertz metamaterial absorber based on Pythagorean-tree fractal geometry,"Optical Materials Express, vol. 10, no. 12, p. 3007, 2020.

17. J. Wang, T. Lang, T. Shen, C. Shen, Z. Hong, and C. Lu, “Numerical study of an ultra-broadband all-silicon terahertz absorber," Appl. Sci., vol. 10, no. 2, p. 436, Jan. 2020.

18. B.-X. Wang, C. Tang, Q. Niu, Y. He, and R. Chen, "A broadband terahertz metamaterial absorber enabled by the simple design of a rectangular-shaped resonator with an elongated slot," Nanosc. Adv., vol. 1, no. 9, pp. 3621-3625, Sep. 2019, doi: 10.1039/c9na00385a.

19. M. Rahmanzadeh, H. Rajabalipanah, and A. Abdolali, "Multilayer graphene-based metasurfaces: Robust design method for extremely broadband, wide-angle, and polarization-insensitive terahertz absorbers," Appl. Opt., vol. 57, no. 4, p. 959, Feb. 2018, doi: 10.1364/ao.57.000959.

20. H.-F. Zhang, J.-X. Liu, J. Yang, H. Zhang, and H.-M. Li, "A polarization insensitive broadband terahertz absorber with a multilayer structure," Results Phys., vol. 11, pp. 1064-1074, Dec. 2018, doi: 10.1016/j.rinp.2018.11.010.

21. H. Liu, Z.-H.Wang, L. Li, Y.-X. Fan, and Z.-Y. Tao, “Vanadium dioxide assisted broadband tunable terahertz metamaterial absorber," Sci. Rep., vol. 9, no. 1, pp. 1-10, Dec. 2019, doi: 10.1038/s41598-019-42293-9.

22. C. Shi et al., "Compact broadband terahertz perfect absorber based on multi-interference and diffraction effects," IEEE Trans. THz Sci. Technol., vol. 6, no. 1, pp. 40-44, Jan. 2016, doi: 10.1109/TTHZ.2015.2496313.

23. G. Deng, J. Yang, and Z. Yin, “Broadband terahertz metamaterial absorber based on tantalum nitride," Appl. Opt., vol. 56, no. 9, p. 2449, Mar. 2017, doi: 10.1364/ao.56.002449.

24. X. Liu, Q. Zhang, and X. Cui, “Ultra-broadband polarization independent wide-angle THz absorber based on plasmonic resonances in semiconductor square nut-shaped metamaterials," Plasmonics, vol. 12, no. 4, pp. 11371144, Aug. 2017, doi: 10.1007/s11468-016-0368-1.

25. J. Yang et al., "Broadband terahertz absorber based on multi-band continuous plasmon resonances in geometrically gradient dielectric loaded graphene plasmon structure," Sci. Rep., vol. 8, no. 1, pp. 1-8, Dec. 2018, doi:

10.1038/s41598-018-21705-2. 
26. G. Varshney, “Wideband THz Absorber: By Merging the Resonance of Dielectric Cavity and Graphite Disk Resonator,"IEEE Sensors Journal, vol. 21, no. 2, pp. 1635-1643, 2021.

27. G. Varshney, "Reconfigurable graphene antenna for THz applications: A mode conversion approach," Nanotechnology, vol. 31, no. 13,Mar. 2020, Art. no. 135208, doi: 10.1088/1361-6528/ab60cc.

28. M. Walther, D. G. Cooke, C. Sherstan, M. Hajar, M. R. Freeman, and F. A. Hegmann, "Terahertz conductivity of thin gold films at the metal insulator percolation transition,” Phys. Rev. B, Condens. Matter, vol. 76, no. 12, pp. 1-9, Sep. 2007, doi: 10.1103/PhysRevB.76.125408.

29. X.-R. Kong, H.-F. Zhang, and R.-N. Dao, "A tunable ultra-broadband THz absorber based on a phase change material," J. Electron. Mater., vol. 48, no. 11, pp. 7040-7047, Nov. 2019, doi: 10.1007/s11664-019-07511-0.

30. T. K. Nguyen, P. T. Dang, I. Park, and K. Q. Le, “Broadband THz radiation through tapered semiconductor gratings on high-index substrate," J. Opt. Soc. Amer. B, Opt. Phys., vol. 34, no. 3, pp. 583-589, 2017, doi:

10.1364/josab.34.000583

31. A. Alù, G. D’Aguanno, N. Mattiucci, and M. J. Bloemer, “Plasmonic brewster angle: Broadband extraordinary transmission through optical gratings," Phys. Rev. Lett., vol. 106, no. 12, pp. 1-4, Mar. 2011, doi:

10.1103/PhysRevLett.106.123902.

32. M. Amin, M. Farhat, and H. Ba` gcl, “An ultra-broadband multilayered graphene absorber," Opt. Express, vol. 21, no. 24, p. 29938, Dec. 2013, doi: 10.1364/oe.21.029938

33. A. Fardoost, F. G. Vanani, A. Amirhosseini, and R. Safian, "Design of a multilayer graphene-based ultrawideband terahertz absorber," IEEE Trans. Nanotechnol., vol. 16, no. 1, pp. 68-74, Jan. 2017, doi: 10.1109/

TNANO.2016.2627939

34. J. Yang et al., "Broadband terahertz absorber based on multi-band continuous plasmon resonances in geometrically gradient dielectric loaded graphene plasmon structure," Sci. Rep., vol. 8, no. 1, pp. 1-8, Dec. 2018, doi:

$10.1038 /$ s41598-018-21705-2.

\section{Figures}

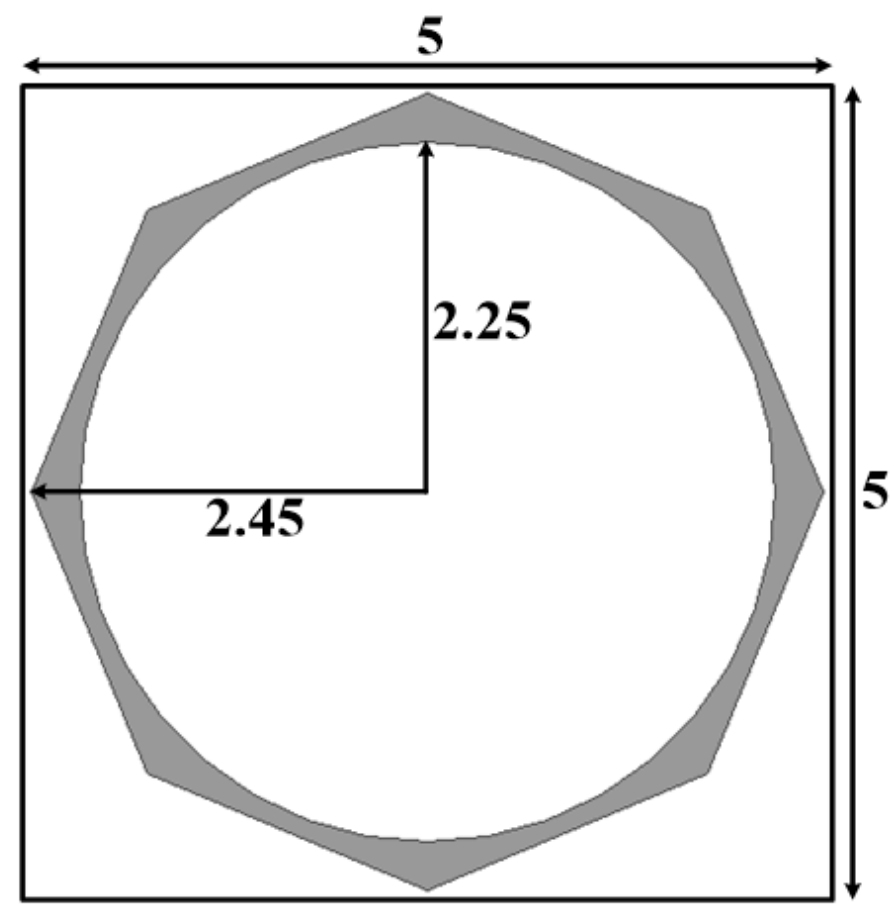

(a)

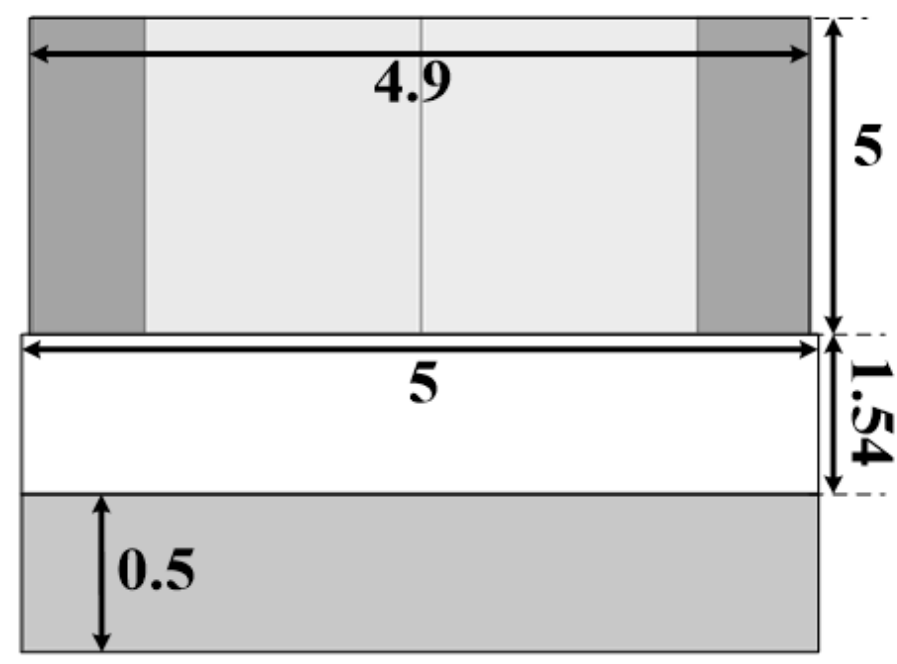

(b) 


\section{Figure 1}

Geometry of proposed absorber (a) top view, (b) side view (all dimensions are in $\mu \mathrm{m}$ )

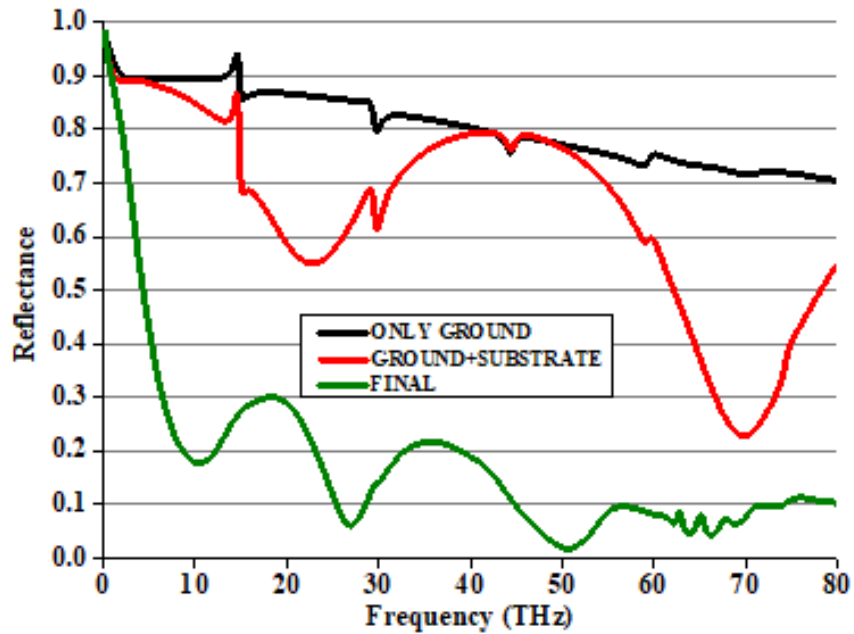

(a)

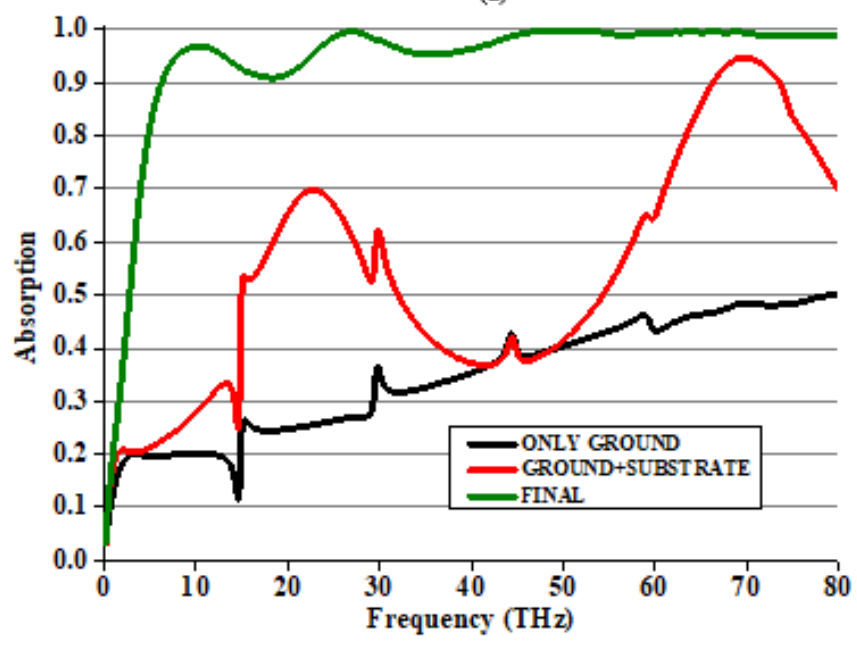

(c)

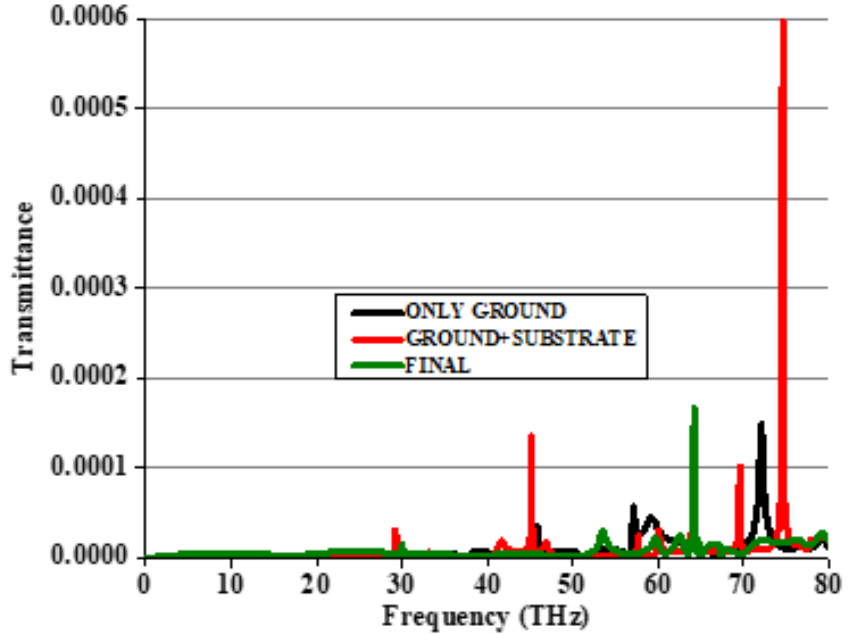

(b)

Figure 2

Performance of design stages (a) reflectance magnitude, (b) transmittance magnitude and (c) absorption 


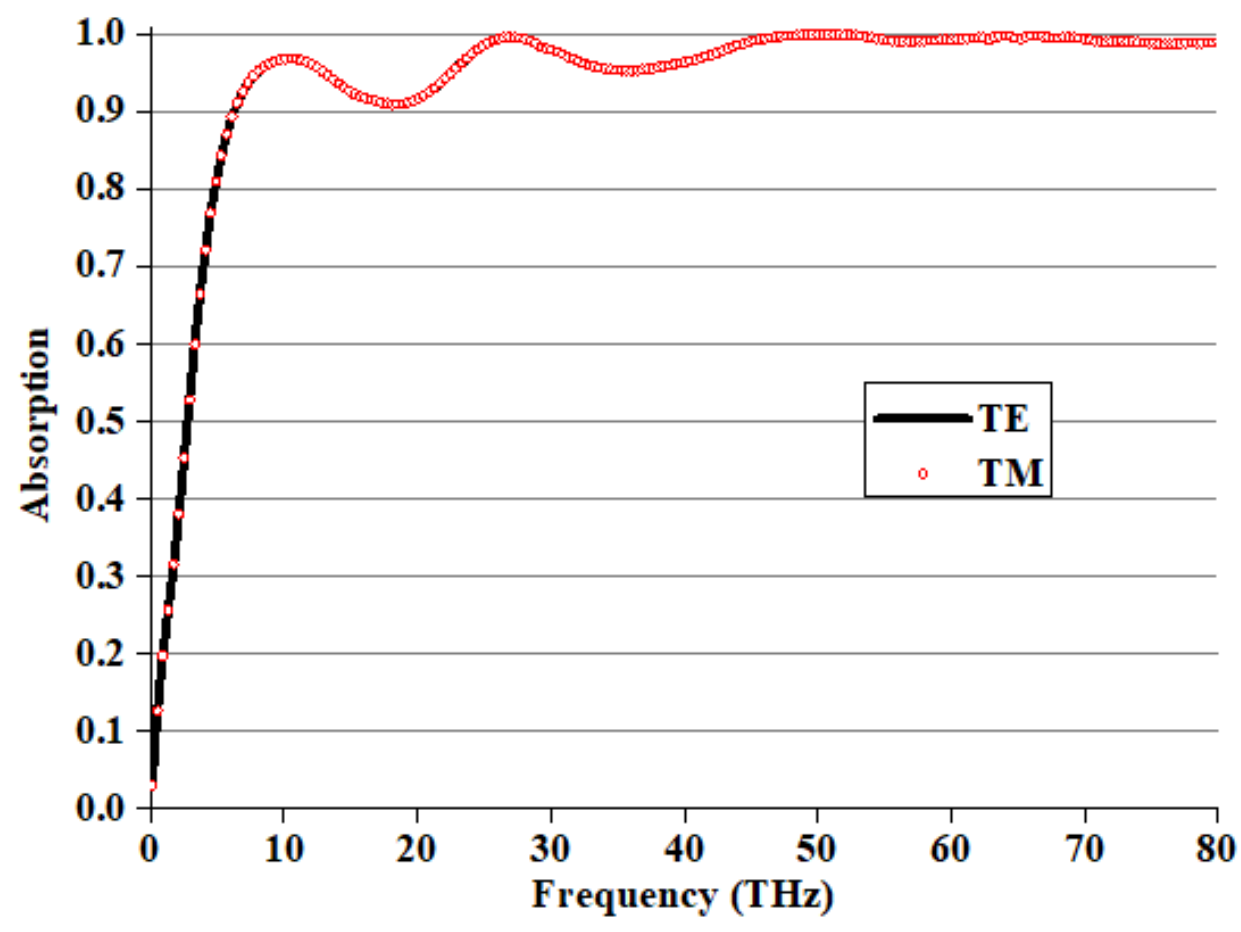

Figure 3

Absorption in TE and TM modes

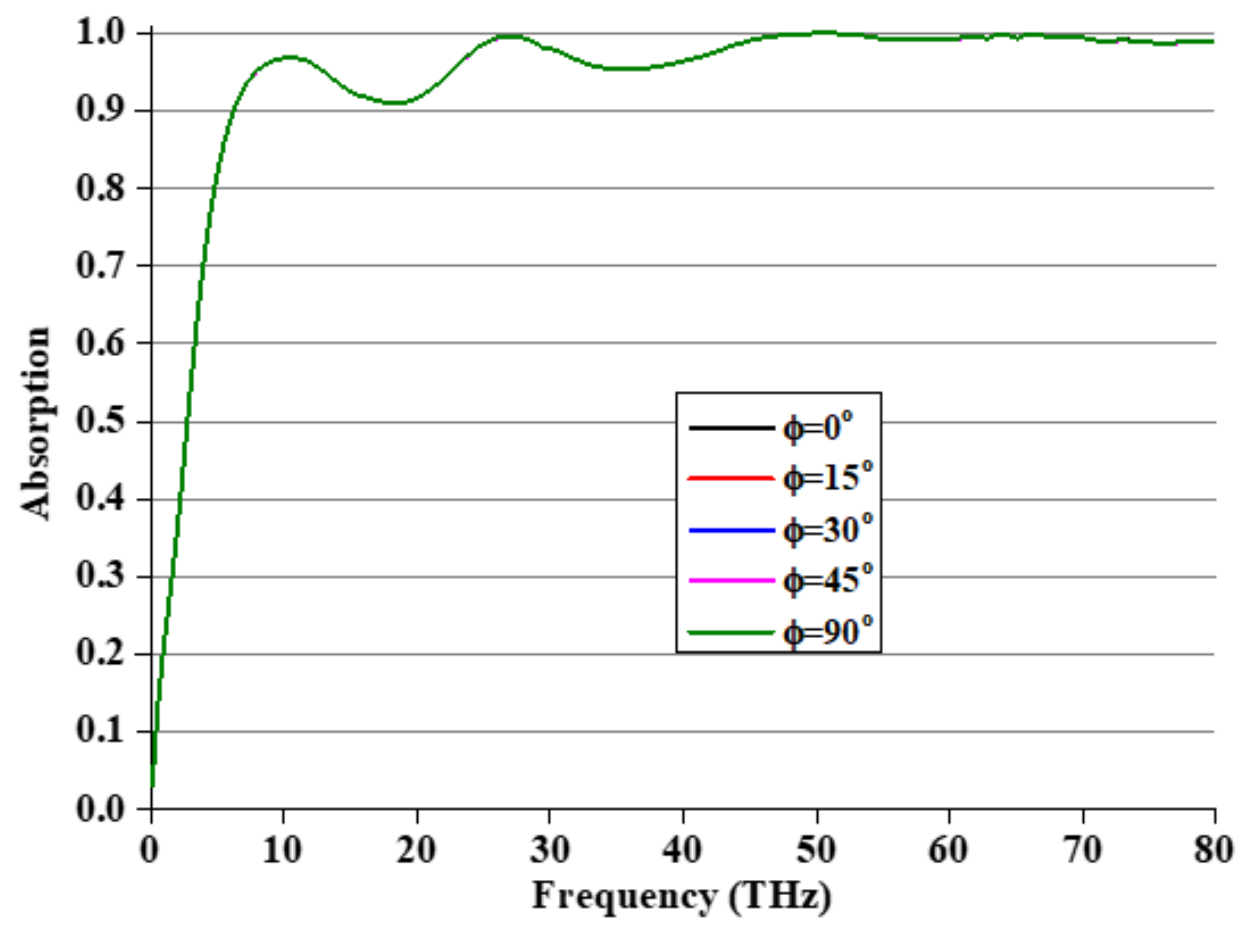

Figure 4

Absorption of proposed absorber with variations in polarization angle 


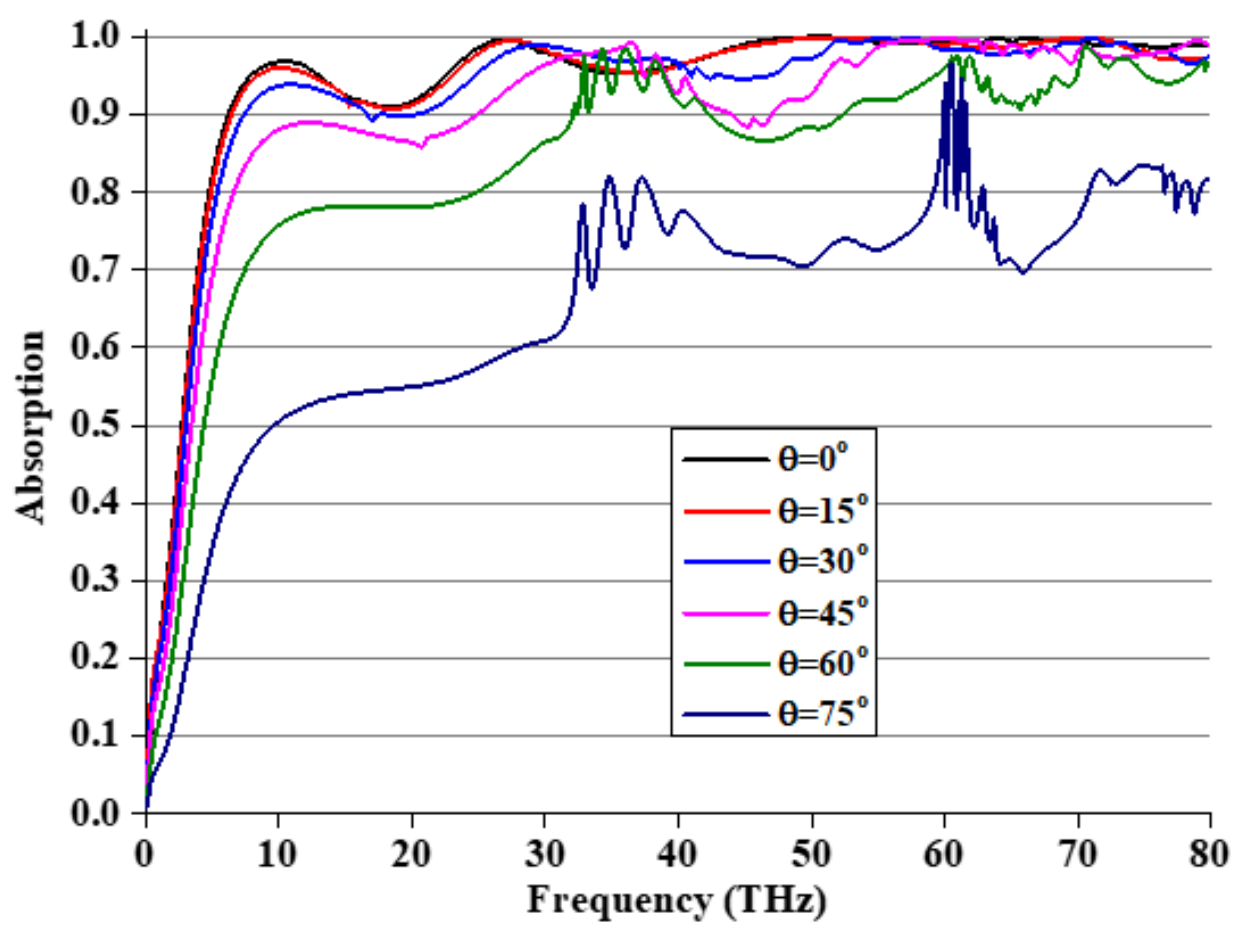

Figure 5

Absorption of proposed absorber with variations in incidence angle in TE mode

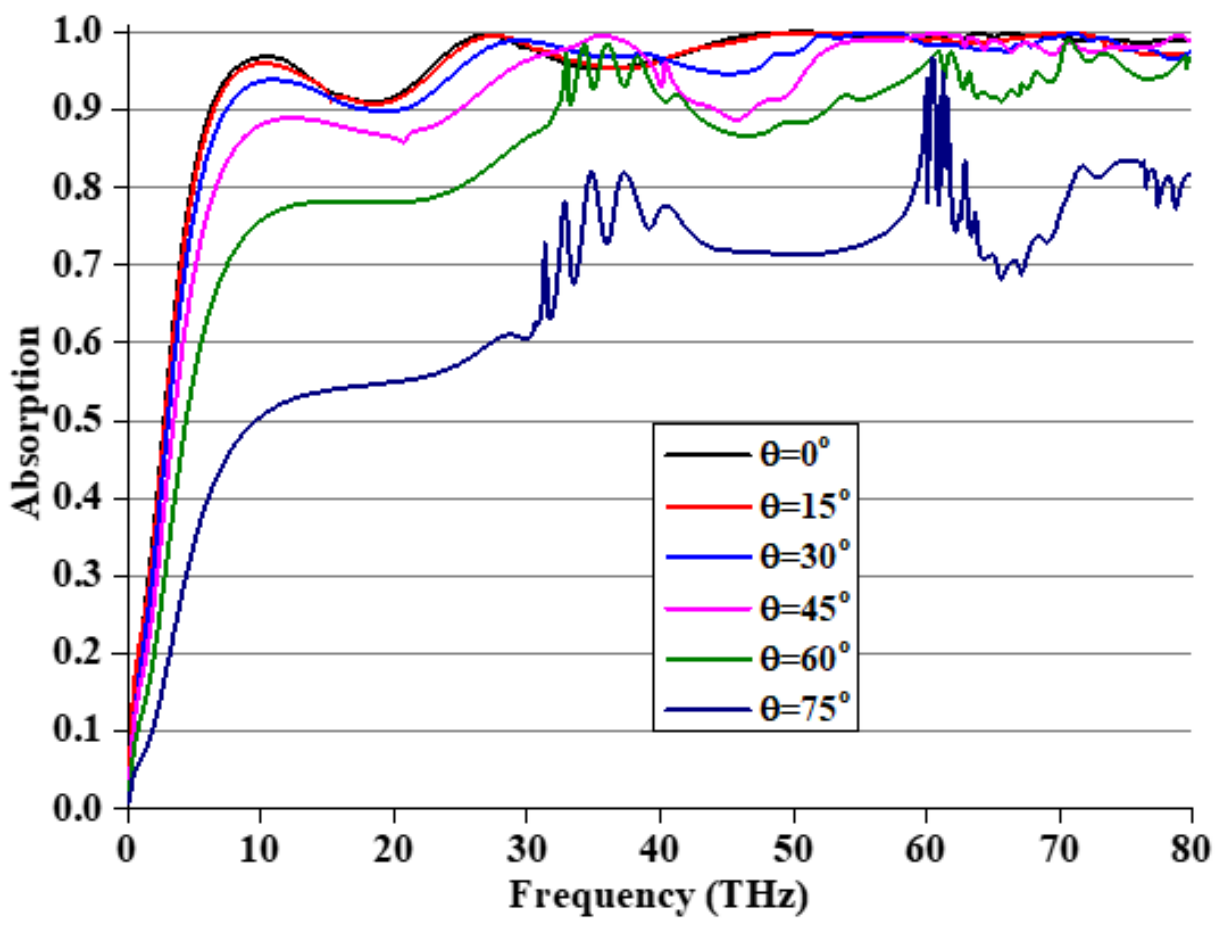

Figure 6

Absorption of proposed absorber with variations in incidence angle in TM mode 